\title{
A cadaveric study of common carotid arteries for the presence of additional branches
}

\author{
Premakumari C. R. ${ }^{1}$, Dnyaneshwar ${ }^{2, *}$ \\ ${ }^{\mathbf{1}}$ Assistant Professor, Karwar Institute of Medical Sciences, Karwar, Karnataka, ${ }^{2}$ Associate Professor, Dept. of Anatomy, Koppal \\ Institute of Medical Sciences, Koppal, Karnataka, India
}

*Corresponding Author:

Email: drdnyan83@gmail.com

\begin{abstract}
Introduction: The common carotid arteries with their terminal branches provide the major source of arterial blood to the head, neck and brain. Among the arterial branches in the human body, carotid bifurcation is particularly important to avoid fatal mix up with internal carotid artery which supplies brain. A profound knowledge of variations in branching pattern is essential during carotid artery catheterization, carotid endartectomy and in thyroid surgeries to avoid fatal complications.

Aims and Objectives: Present study was carried out to observe the origin of additional branches from common carotid arteries, excluding the terminal branches. i.e. internal and external carotid arteries

Material and Methods: The present study was carried out in a total of 54 common carotid artery specimens, dissected in 27 embalmed cadavers (23 males, 4 females). Out of these, 27 left CCAs and 27 right CCAs were studied in the department of Anatomy, J.J.M Medical college, Davangere, India. The specimens were studied by detailed dissection method.

Results: In the present study, $51.9 \%$ specimens showed additional branches; superior thyroid artery being the commonest $(40.7 \%)$.Trifurcation, with the origin of ascending pharyngeal artery at the termination was observed in $1.9 \%$ of the common carotid arteries.

Conclusions: In the present study, frequent origin of superior thyroid artery from common carotid artery at different levels was found. The incidence of STA origin was higher compared to other studies. Origin of inferior thyroid artery from common carotid artery is the rarest possibility. In the present study, two specimens (3.7\%) showed the origin of ITA from CCA.
\end{abstract}

Keywords: Common carotid artery, Superior thyroid artery, Inferior thyroid artery, Trifurcation.

\section{Introduction}

The common carotid arteries (CCA) with their terminal branches internal and external carotid arteries provide the major source of blood to head and neck. External carotid supplies the exterior of head, face and most of the neck and internal carotid supplies cranial and orbital contents. CCA differ on the right and left sides with respect to their origins. On the right, the CCA arises from brachiocephalic trunk as it passes behind the sternoclavicular joint. While it's a direct branch of the arch of aorta on the left. The right CCA therefore has only cervical part and left CCA has both cervical and thoracic parts. Following same course on both left and right sides, the CCA bifurcates at the level of upper border of thyroid cartilage (C3-C4 junction) into external and internal carotid arteries. Artery can be compressed against Chassaignac's tubercle opposite C6 level. Variants in origin, course and termination of common carotid artery is of great clinical value during head and neck surgeries and angiographies to avoid a fatal mix up of the internal carotid artery which supplies brain. The origin and course of superior thyroid and inferior thyroid arteries originating as a variations from the common carotid arteries could be of interest in thyroid surgeries. The study may be useful for vascular surgeons in various head and neck surgeries, angiographies and other intravascular interventions involving the common carotid arteries. ${ }^{1}$

\section{Material and Methods}

The study was carried out in a total of 54 CCA specimens from 27 well embalmed cadavers (23 males, 4 females) alloted for routine undergraduate dissection, in the department of Anatomy, J.J.M Medical College, Davangere, Karnataka, India for a period of three years. The age of both sexes were approximately 60 to 75 years.

Ethical clearance from the institution was obtained \& methodology prescribed by Cunningham's Manual of Practical Anatomy was followed.

\section{Results}

Among the 54 specimens studied, one specimen exhibited trifurcation of CCA, dividing into external carotid, internal carotid and ascending pharyngeal arteries. The remaining 53 showed the normal pattern of termination, with the CCA bifurcating into external and internal carotid arteries. Out of the 54 specimens, 28 showed additional branches of which $40.7 \%$ [22] were superior thyroid artery, \% [2] were inferior thyroid artery and \% 2 were lingual artery. Superior laryngeal artery and ascending pharyngeal artery, were $2 \%$ each. $^{1}$ 
Table 1

\begin{tabular}{|l|c|c|}
\hline $\begin{array}{c}\text { Additional } \\
\text { branches of CCA }\end{array}$ & $\begin{array}{c}\text { No.of specimens } \\
(\mathbf{N = 5 4})\end{array}$ & Percentage \\
\hline STA & 22 & 40.7 \\
\hline ITA & 2 & 3.7 \\
\hline SLA & 1 & 1.9 \\
\hline LA & 2 & 3.7 \\
\hline APA & 1 & 1.9 \\
\hline Total & 28 & 100 \\
\hline
\end{tabular}

\section{Discussion}

A number of variations of CCA are of interest. The artery may occasionally give rise to the superior thyroid, superior laryngeal, ascending pharyngeal, inferior thyroid or occipital arteries. ${ }^{1}$

The above variations may have embryological basis. The CCAs arise from an elongation of the aortic sac and the third arch artery becomes the proximal part of ICA. The ECA arises as a sprout from the common carotid. ${ }^{1}$ The fourth aortic arches leads to development of arch of aorta and left subclavian arteries. Since ECA arises as a part of CCA, there are chances that branches of ECA could have direct origin from CCA or from ICA. Occasionally branches of subclavian artery may arise from CCA because of close approximation of proximal parts of third and fourth aortic arches.

The present study included 54 common carotid specimens dissected in 27 embalmed cadavers which were studied for any additional branches apart from external and internal carotid arteries. In the present study additional branches of common carotid arteries were found in 28 specimens out of 54 specimens. Superior thyroid artery originated from common carotid artery in 22 specimens $(40.7 \%)$ while the Inferior thyroid artery originated in 2 specimens $(3.7 \%)$. Superior laryngeal artery originated from common carotid artery in 1 specimen $(1.9 \%)$. Lingual artery in 2 specimens $(3.7 \%)$ and ascending pharyngeal artery in 1 specimen $(1.9 \%)$ originated from common carotid artery.

A study by Chitra about the carotid system of arteries for a period of three years showed the origin of right occipital artery at carotid bifurcation in a male cadaver and the APA was the branch of right internal carotid artery. ${ }^{2}$

A study by Natsis $\mathrm{K}$ in 100 common carotid specimens showed that STA originated from CCA in $61 \%$ of cases. ${ }^{3}$ Lo A studied 67 CCA specimens out of which STA originated from CCA in $52.3 \%$ and $46.2 \%$ originated from ECA. ${ }^{4}$ In a study of sixty CCA in 30 cadavers, in $18.3 \%$ of cases, STA originated from CCA. ${ }^{5}$

Unusual case of origin lingual artery from CCA 6 $\mathrm{mm}$ below the level of bifurcation has been reported. ${ }^{6}$ Babu, ${ }^{7}$ Budhiraj and Rastogi reported origin of thyrolingual trunk from right and left CCA respectively. ${ }^{8}$
A rare case has been reported in which there was combined high bifurcation of the $\mathrm{CCA}$, an anomalous origin of APA from CCA and unusual branching pattern of external carotid artery. ${ }^{9}$

Menezes FO reported the APA and inferior thyroid artery originating from the common carotid artery respectively. ${ }^{10}$

A study by Mohan das over the carotid system of arteries found that STA was arising from CCA about 1 $\mathrm{cm}$ proximal to its bifurcation. The subsequent course of STA was found to be normal. ${ }^{11}$ A cadaveric study on origin of STA was done in a Kenyan population observed the origin of STA from CCA in $13 \%$ of cases. Origin from the left CCA was associated with high carotid bifurcation. $^{12}$

A rare case of a left middle thyroid artery arising from CCA was reported in a 71 year old male cadaver. The right and left STA arose from CCA in the same cadver. ${ }^{13}$

An anomalous artery directly connecting CCA and ICA was discovered on the right side in the cadaver of a 83 year old female and the facial artery, maxillary artery and superficial temporal artery arise from the common carotid by a common trunk. ${ }^{14}$ A case was reported in which thyrolingual trunk originated from CCA in a 73 year old female as revealed by computed tomography angiography for vascular mapping of the carotid vessels before head and neck microsurgical reconstruction. ${ }^{15}$ As compared to other studies our study showed the higher incidence of STA and ITA from CCA.

Origin of STA from CCA on the left $1 \mathrm{~cm}$ proximal to its bifurcation has been reported by Mohandas. Though the artery arise from CCA, it accompanied external Laryngeal Nerve as usual and followed normal course. $^{16}$

Deficiency (lacunae) of the present study can be stated that the study would have been still more useful if the common carotid arteries were studied by various other methods along with cadaveric study.

\section{Conclusion}

The following factors were concluded from the study: In the present study the frequent origin of STA from CCA at different levels was found.

Superior thyroid artery was the commonest additional branch from CCA in the present study. The incidence being higher in the present study compared to other studies.

Origin of inferior thyroid artery from $\mathrm{CCA}$ is a rarest possibility. In the present study 2 specimens (3.7\%) showed the origin of ITA from CCA . The incidence is significantly higher in present study compared to other studies. 
Lingual artery originated from CCA in 2 specimens (3.7) which is also of a rare variation.

The study will be useful for surgeons during various surgeries which involve CCA as well as its additional branches to avoid fatal complications.

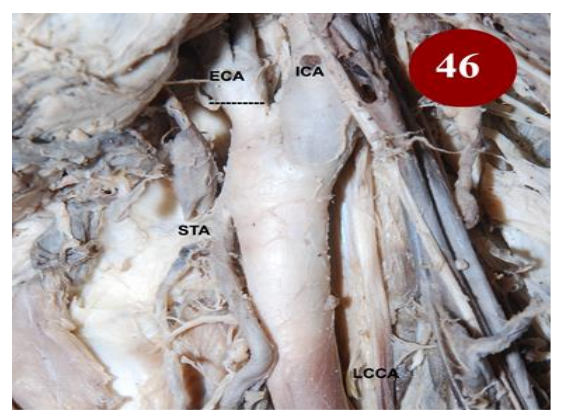

Fig. 1: STA arising from LCCA $2 \mathrm{~cm}$ below bifurcation

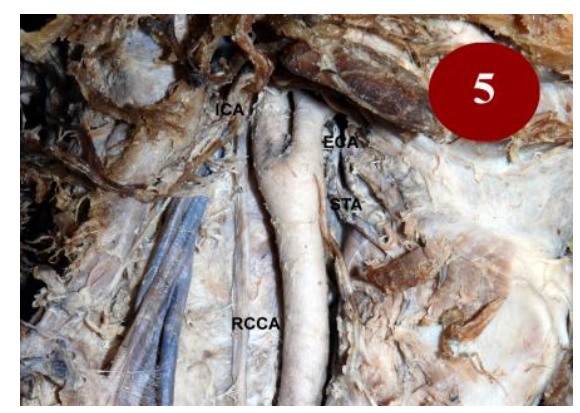

Fig. 2: STA arising from RCCA $0.5 \mathrm{~cm}$ below bifurcation

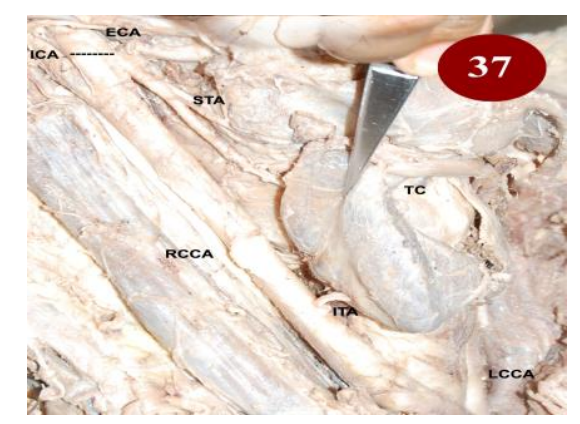

Fig. 3: ITA arising from RCCA $5 \mathrm{~cm}$ above origin of RCCA

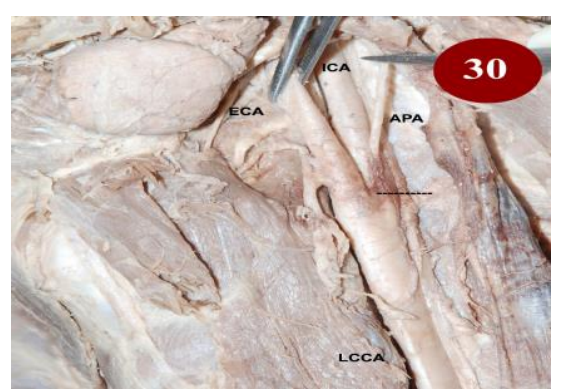

Funding: No funding sources.

Conflict of interest: None declared.

\section{References}

1. Susan Standring ed. Gray's anatomy. The anatomical basis of clinical practice. $40^{\text {th }}$ ed. London: Churchill Livingstone Elsevies 2008:453,610.

2. R. Chitra. Trifurcation of the right common carotid artery- case report. Indian J Plast Surg 2008;41(1):85-7.

3. Natsis K, Raikos A, Foundos I, Noussisos G, Lazaridis N, Njau SN.Superior thyroid artery origin in Caucasian greeks: A new classification proposal and review of the literature. Clin Anat 2011;24(6):699-705.

4. Lo A, Oehley M, Bartlett A, Adams D, Blyth P, Ali SA. Anatomical variations of the common carotid bifurcation. ANZ J Surg 2006:76(11):970-72.

5. Rafiah AA, Haggagy AAE, AalbIHA, Zaki AL. Anatomical study of the carotid bifurcation and origin variations of the ascending pharyngeal and superior thyroid arteries. Folia Morphol(Warz) 2011;70(1):47-95.

6. Jadhav SD, Ambali MP, Patil RJ. Anatomical variation of the origin of the right lingual artery-case report. Int $J$ Anat Var 2011;4:75-76.

7. Babu BP. Anomalous origin of thyrolingual trunk from right common carotid artery-A case report. J Anat Soc India 2001;50(1):47.

8. Budhiraja V, Rastogi R. Variant origin of thyrolingual trunk from Left common carotid artery- case report. Int $J$ Anat Var 2010;3:44-5.

9. Gluncic V, Petanjek Z, Marusic A, Gluncic I.High bifurcation of common carotid artery, anomalous origin of ascending pharyngeal artery and anomalous branching pattern of external carotid artery. Surg and Radiol Anat 2001;23(2):123-25.

10. Menezes FO, Demartini JZ, Matos LA, Godoy JM, Spotti AR, Sants ML. Anomalous branch of the right common carotid artery. Arq Neuropsiquiatr 2008;66(4):916-17.

11. Mohandas KGR, Asutosh SR. Unusual origin of the arterie in the carotid triangle of the neck: A case report and literature review. Open Anat J 2009;1:24-7.

12. Gurbuz J, Cavdr S, Ozdogmus O. Trifurcation of the left common carotid artery. A case report. Clin Anat 2001;14:58-6.

13. Ongeti KW, Ogengo JA. Variant origin of the superior thyroid artery in a Kenyan population. Clin Anat 2011;24(7):797-99

14. Won HS,Han SH,OH CS,Chung JH.Superior and middle thyroid arteries arising from the common carotid artery. Surg Radiol Anat 2011;50(4):23-5.

15. Limura A, Oguchi T, Yamazaki Y, Matsua M, Yi SQ, Itoh $\mathrm{M}$ et al. Anomalous bifurcation and island formation of the carotid artery. Okajimas Folia Anat Jpn 2010:101:91-3.

16. Smit SD, Benton RS.A rare origin of the superior thyroid artery. Acta Anatomica 1978;101:91-3.

17. Mohandas KGR, Asutosh SR. Unusual origin of the arteries in the carotid tringle of the neck: A case report and literature review. Open Anat J 2009;1:24-7. 

Abbreviations
APA: Ascending pharyngeal artery
BCT: Brachiocephalic trunk
CCA: Common carotid artery
ECA: External carotid artery
ICA: Internal carotid artery
ITA: Inferior thyroid artery
LA: Lingual artery
LCCA: Left common carotid artery

RCCA: Right common carotid artery

STA: Superior thyroid artery

TC: Thyroid cartilage

How to cite the article: R. Premakumari, Dnyaneshwar. A cadaveric study of common carotid arteries for the presence of additional branches. Indian J Anat Surg Head Neck Brain 2018;4(3):65-68. 